\title{
Representación y magnitud de los sumandos en la resolución de problemas aditivos
}

\author{
VICENTE BERMEJO \\ MARÍA OLIVA LAGO \\ Universidad Complutense de Madrid
}

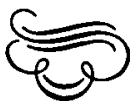

Resumen

Se estudia la incidencia del nivel de abstracción y de la magnitud de los sumandos en la resolución de tareas aditivas elementales. Para ello, dos muestras de niños de 5 a 7 años pasaron diferentes pruebas. Los resultados muestran en general que los factores edad, modo de representación y el tamaño y posición de los sumandos son estadisticamente significativos. Más en concreto, los niños pequeños son especialmente sensibles al grado de abstracción de los sumandos, mientras que el grupo de los mayores se ve afectado principalmente por el tercer factor. Por otra parte, el repertorio de estrategias empleado es pobre en los preescolares y variado en los escolares.

Palabras clave: Desarrollo, conceptos aritméticos, resolución de problemas de adición, representación de la suma.

\section{Representación and size of the addends in solving addition problems}

\section{Abstract}

In this paper we studied the effects of the abstraction level and the addend size on the solution of elemental addition tasks. To this end, different tests were presented to two arrays of children aged from 5 to 7 . The data show, in general, that factors like age, type of representation, and size and placing of the addends are statistically significant. More specifically, the younger children are specially sensitive to the abstraction level of the addends, while the group of older children is mainly affected by the third factor. On the other hand, the type of strategies employed is quite poor in the of preschool group and diverse in the elementary school groups.

Keywords: Development, Arithmetical Concepts, Addition Problem-Solving, Addend Representation.

Agradecimientos: Algunas de las ideas de este trabajo están recogidas en una comunicación a las II Jornadas Internacionales de Psicología y Educación, Madrid, junio de 1986.

Dirección del autor: Universidad Complutense de Madrid, Facultad de Psicología, Departamento de Psicología Evolutiva, Campus de Somosaguas. 28071 Madrid.

Original recibido: Diciembre 1987. Revisión recibida: Septiembre 1988. Aceptado: Septiembre 1988 
Entre las múltiples investigaciones que se han llevado a cabo en torno a la adquisición de las nociones aritméticas elementales, ocupa un lugar central el estudio de la adición y sustración, siendo numerosos tanto los trabajos que se refieren a la ejecución de los algoritmos de suma y resta como los relativos al modo en que tales algoritmos se aplican a la resolución de problemas. Dentro del primer tipo de investigaciones, destacan los que utilizan modelos de simulación para obtener un diagnóstico adecuado de los errores y poder así contribuir a su corrección (Brown y Burton, 1978; Brown y VanLehn, 1980, 1982; Young y O'Shea, 1981). Sin embargo, como muestran Brown y Campione (1986), existe aún un notable salto desde las formulaciones teóricas al campo de la instrucción, siendo precisos no pocos esfuerzos que busquen la congruencia de ambos criterios.

Dentro del segundo grupo, relativo a la resolución de problemas aritméticos elementales, son numerosos los estudios que intentan determinar la relevancia de los diversos factores intervinientes (Bermejo y Lago, en prensa; Bermejo y Rodríguez, en prensa; Carpenter y Moser, 1982, 1983; De Corte y Verschaffel, 1985; Riley, Greeno y Heller, 1983, etc.). Así, por ejemplo, Riley et al. (1983) diferencian entre factores globales y factores específicos, refiriéndose estos últimos a las características estructurales de las oraciones de los problemas, a la habilidad de lectura, a la repercusión del método de instrucción seguido y, sobre todo, a la presencia de ayudas en el momento de dar solución a un problema. Con respecto a la influencia de las ayudas, dichos autores consideran que la presencia de objetos manipulables conduce a una mejora en la ejecución de los niños, siendo incluso necesaria en algunos casos, como se desprende de los Modelos I y II propuestos para explicar los estadios de conocimiento conceptual requerido para la resolución de diversos tipos de problemas.

Igualmente, otros estudios también ponen de relieve el efecto facilitador de la presencia de ayudas (por ejemplo: fichas, bloques, etc.) durante la resolución de los problemas, de tal modo que estos efectos aparecen tanto cuando se trata de una mera observación de las mismas, sin manipularlas (por ejemplo, Buckingham y MacLatchy, 1930; Gibb, 1965), como cuando se manipulan (Carpenter, Hiebert y Moser, 1983; Carpenter y Moser, 1982; Steffe y Johnson, 1971). En efecto, Carpenter et al. (1983) destacan que la utilización de bloques simplifica la resolución de los problemas planteados a los niños de primer grado. En un estudio posterior, Carpenter y Moser (1982) especifican la importancia de las ayudas en la resolución de problemas aditivos cuando el tamaño de los sumandos es elevado. Steffe y Johnson (1971) también observan en diversos tipos de problemas de cambio y combinación que los resultados son mejores ante la presencia de objetos que en ausencia de los mismos. Pero quizá la incidencia de la presencia de objetos en la resolución de problemas aritméticos elementales sean aún más notable en torno a la etapa prenumérica, durante la cual se hace patente la necesidad de apoyos externos de representación (Steffe, Thompson y Richards, 1982). El trabajo de Riley (1981), citado por Riley et al., 1983) pone asimismo de manifiesto que los niños de preescolar son más eficientes ante 4 de los 6 tipos de problemas de cambio presentados cuando disponen de ayudas; en los restantes no es así debido la excesiva facilidad o dificultad de los problemas propuestos.

Un aspecto menos estudiado, pero no por ello de menor importancia, 


\section{1}

se refiere al tipo de representación empleado en la resolución de los problemas. A pesar de que algunos trabajos lo tienen presente, sin embargo, no constituyen su objeto prioritario de estudio, por lo que continúa siendo un tema capital en la polémica de cómo mediar en la enseñanza de las primeras nociones aritméticas y de cómo facilitar el tránsito hacia la utilización comprensiva de los símbolos aritméticos. No obstante, algunas investigaciones (por ejemplo, Fuson, 1982, 1984, 1986; Jones, Thornton y Toohey, 1985; Secada, Fuson y Hall, 1983) comienzan a ocuparse de ciertas situaciones específicas. Así, Fuson (1986) considera que la utilización simultánea de materiales concretos y abstractos resulta bastante efectiva en la instrucción de la estrategia de contar a partir de un sumando. También, Secada et al. (1983) concluyen que ese tipo de materiales (así como las preguntas utilizadas en su diseño) parecen organizar el conocimiento de algunos niños y facilitar el cambio hacia la estrategia más avanzada de contar a partir de uno de los sumandos. En esta misma línea, Jones et al. (1985), dentro del programa multiopción para el aprendizaje de automatismos numéricos, emplean en el principio del mismo una secuencia con cinco pasos (acción física; gráfica; acción-gráfica; representación unitaria de la adición; y formulación estándar), progresivamente más abstractos, para allanar la transición a una estrategia más evolucionada de «count-on». Esta estrategia sirve de trampolín hacia un nivel más eficiente basado en el recuerdo de combinaciones numéricas. Los citados autores valoran positivamente la utilización de una secuencia cada vez más abstracta, basada en la utilización conjunta de elementos concretos y símbolos, para que el niño llegue a un método de resolución más sofisticado que se apoye principalmente en la secuencia de numerales. Y en esta línea, Case $(1978,1982)$ subraya la importancia de graduar el nivel de abstracción para obtener una mayor automatización en las ejecuciones de los niños, antes de introducir elementos más abstractos.

Otro factor específico en el proceso de resolución de un problema es la magnitud de los sumandos. Este aspecto fue el tema preferido de las primeras investigaciones acerca de las capacidades aritméticas de los niños, de modo que Knight y Behrens (1928), por ejemplo, advierten que cuanto mayor es el resultado, más difícil resulta el problema. Recientemente aparecen estudios cronométricos, que proponen modelos y datos que hacen mención principalmente a los efectos de varios factores: el sumando mayor, el tamaño del segundo sumando, la suma al cuadrado, las redes almacenadas de combinaciones o el conocimiento de procedimiento regido por diversos principios (Ashcraft, 1982, 1983; Baroody, 1983; Groen y Parkman, 1972; Groen y Poll, 1973; Suppes y Groen, 1967), así como a la mayor facilidad de los dobles (Groen y Parkman, 1972; Svenson, 1975; Svenson y Broquist, 1975).

Algunas investigaciones muestran que cuando el cardinal de los sumandos es superior a 10, entonces se utiliza más frecuentemente la estrategia de "count-on", que ante sumandos inferiores a 10 (Moser, 1980, citado por Secada et al., 1983); o que dicha estrategia se utiliza más ampliamente cuando el primer sumando toma valores superiores a 15 que cuando se encuentra entre 12 y 15 (Secada y Fuson, citados por Secada et al., 1983). En este mismo sentido deben considerarse los resultados de Siegler y Robinson 


\section{2}

(1982), cuando indican que las estrategias empleadas por los niños son función de la magnitud de los sumandos, así como que la dificultad de un problema crece monótonamente a medida que los sumandos van tomando valores de uno a cuatro, tendencia que no se mantiene cuando cualquiera de ellos es cinco. Otro ejemplo del efecto del tamaño de los sumandos lo proporcionan Carpenter y Moser (1982) cuando crean diversas condiciones para la presentación de problemas aditivos en función del tamaño de las cantidades del problema y de la presencia o no de ayudas. Estos autores encuentran que los niños pueden resolver antes los mismos problemas con valores reducidos que con valores más elevados. Por otra parte, consideran especialmente importante el comportamiento de los niños cuando los valores de las cantidades son elevadas y disponen de ayudas, ya que en tales ocasiones optan por estrategias más avanzadas de las que se emplearía en caso de que tuvieran ayudas, tal como, por otra parte, encuentran Siegler y Robinson (1982). En definitiva, el tamaño de los sumandos no sólo incide sobre la mayor o menor dificultad de un problema, sino fundamentalmente sobre el tipo de estrategia de resolución elegida por los niños.

El presente trabajo pretende calibrar el alcance de dos factores fundamentales, a nuestro juicio, en la solución de problemas aditivos elementales, que están estrechamente relacionados entre sí. Por una parte, se trata de precisar cómo y cuándo incide especialmente el modo de representar cada uno de los sumandos del problema. Y para ello, planteamos un conjunto de condiciones que varían gradualmente su nivel de abstracción, pasando de la representación más concreta, formada por objetos manipulables, a la más abstracta, cuando los dos sumandos se simbolizan mediante guarismos. Y, por otra, variamos igualmente la magnitud de los sumandos, de modo que el primero de ellos es menor, mayor o igual que el segundo, siendo éste a su vez superior a 10 , menor y mayor que cinco, respectivamente. Así, el niño podrá representarlos mediante los dedos de una mano, de las dos, o no podrá en absoluto, inclinándose quizá en este caso al uso de la propiedad conmutativa, si posee la competencia cognitiva correspondiente. Al mismo tiempo, se estudia detalladamente el tipo de estrategia utilizado por los niños, analizando su relación con el nivel de representación y la magnitud de los sumandos, así como con el grado de éxito alcanzado en la resolución de las pruebas presentadas.

\section{METODO}

Sujetos: Participan en esta investigación 50 niños elegidos al azar en dos colegios nacionales de Madrid de clase sociocultural media. 25 de ellos pertenecen a $2 .^{\circ}$ de preescolar (M: $\left.5 ; 7\right)$ y los otros 25 a $1 .^{\circ}$ de EGB $(\mathrm{M}: 6 ; 7)$. En cada una de las muestras la mitad aproximadamente son niños y el $50 \%$ restante, niñas.

Material: Se emplean fichas de parchís (1 $\mathrm{cm}$ de diámetro) de dos colores, tanto para el pretest, que comprueba el alcance de conteo de los niños, como para la prueba IV. En las cuatro pruebas restantes se utilizan tarjetas $(7,5 \times 10 \mathrm{~cm})$ en las que se representan los sumandos de los problemas planteados, sea mediante círculos rojos $(1 \mathrm{~cm}$ de diámetro) dispuestos verticalmente, sea mediante guarismos. El uso de los muñecos televisivos Epi y Blas permite escenificar los problemas y motivar a los niños. 
Procedimiento: Cada niño pasa las tres tareas aditivas siguientes (A: $5+12 ; \mathrm{B}: 11+4 ; \mathrm{C}: 8+8)$ representando de modo diferente cada uno de los sumandos (I: guarismos + círculos; II: círculos + círculos; III: círculos + guarismos; IV: fichas + fichas; V: guarismo + guarismo), y utilizando en todos los casos problemas de cambio. El orden de cada una de las presentaciones y el de las distintas operaciones dentro de cada una se realiza el azar y es constante para todos los sujetos. Los niños pasan individualmente cada tarea en un total de aproximadamente veinte minutos, $y$ antes de comenzar con los problemas, que constituyen el objetivo del estudio se comprueba el alcance de su secuencia de conteo.

\section{ANALISIS Y DISCUSION DE RESULTADOS}

Los resultados del análisis factorial $(25 \times 2)$ permiten resaltar tres hechos importantes: 1) las diferencias entre los dos grupos de niños son notorias, puesto que el factor edad es estadísticamente significativo $(\mathrm{F}[1,48]=27,7254, \mathrm{p}<.01)$, y estas discrepancias se reflejan tanto a nivel de ensayos correctos como con respecto a la consistencia mostrada en las distintas tareas. 2) La magnitud y ubicación de los sumandos influyen manifiestamente en el comportamiento infantil, siendo igualmente significativos los tres tipos de problemas planteados $(F[2,96]=3,971, p<.05)$. 3) $\mathrm{Fi}$ nalmente, el modo de representar o de simbolizar el cardinal de los sumandos resulta también estadísticamente significativo $(F[4,192]=17,424$, $\mathrm{p}<.01$ ), si bien podemos adelantar ya que, en general, esta variable afecta fundamentalmente a los niños pequeños, siendo menos visibles sus efectos en el grupo de los mayores.

En lo que sigue analizaremos detalladamente cada uno de los tres datos reseñados, si bien el primero lo haremos al mismo tiempo que estudiamos los dos restantes, por razones que, por obvias, no necesitan explicación. Por tanto, con respecto al tipo de problemas propuesto, los niños pequeños encuentran más fácil la prueba $\mathrm{B}(66,4 \%$ de aciertos) que la prueba $\mathrm{C}$ $(63,1 \%)$ y ésta a su vez que la $A(55,2 \%)$. Y en este mismo orden decreciente aparece también el nivel de consistencia en los tres problemas ( $36 \%$, $28 \%$ y $16 \%$, respectivamente). En cambio, el grupo de los mayores presenta un comportamiento relativamente similar en las tres pruebas que resultarían demasiado fáciles para estos sujetos, tal como puede constatarse en las tablas I y II. Su nivel de consistencia es igualmente elevado en las mencionadas tareas, corroborando una vez más su escasa dificultad para estos niños y la ausencia relativa del azar $(68 \%, 84 \%$ y $72 \%$ para A, B y $C$, respectivamente). Las diferencias encontradas en el éxito de los preescolares se debería, a nuestro entender, al menos a dos razones. En primer lugar, el paso de las unidades a la decena supone la actuación de unos mecanismos cognitivos complejos (ver Resnick, 1983) que dificultan lógicamente la ejecución de los niños, tal como ha podido ocurrir en las pruebas A y C. Y, en segundo lugar, el grado de dificultad para representar el segundo sumando sobre todo, que dada su magnitud sería imposible hacerlo con los dedos de una mano (C), o con los de las dos (A), constituye otra de las variables que incrementarían la complejidad de las tareas propuestas. Esta explicación se confirma al observar que la cota de aciertos en los dos 
grupos, pero sobre todo en los preescolares, desciende sensiblemente en aquellas tareas que proponen un guarismo para representar el segundo sumando; lo que sugiere que, al menos en estos casos, los niños utilizan los dedos de la mano, tal como veremos seguidamente.

TABLA I

Porcentajes de aciertos según los distintos tipos de representación y magnitud de los sumandos en los niños de $2 .^{\circ}$ de preescolar

\begin{tabular}{ccccl}
\hline Pruebas & \multicolumn{4}{c}{ Problemas } \\
& $5+12$ & $11+4$ & $8+8$ & Total \\
\hline I & 48 & 60 & 48 & 52 \\
\hline II & 84 & 92 & 87,5 & 87,83 \\
\hline III & 36 & 48 & 44 & 42,67 \\
\hline IV & 80 & 84 & 100 & 88 \\
\hline V & 28 & 48 & 36 & 37,33 \\
\hline TOTAL & 55,2 & 66,4 & 63,1 & \\
\hline
\end{tabular}

TABLA II

Porcentajes de aciertos según los distintos tipos de representación y magnitud de los sumandos en los niños de $1 .^{\circ}$ de $E G B$

\begin{tabular}{rlccl}
\hline Pruebas & \multicolumn{3}{c}{ Problemas } \\
& $5+12$ & $11+4$ & $8+8$ & Total \\
\hline I & 91,67 & 100 & 96 & 93,22 \\
\hline II & 96 & 96 & 96 & 96 \\
\hline III & 88 & 96 & 88 & 90,67 \\
\hline IV & 96 & 92 & 96 & 94,67 \\
\hline V & 84 & 92 & 84 & 86,67 \\
\hline Total & 91,13 & 95,29 & 90,4 & \\
\hline
\end{tabular}

En cuanto al modo de representar los sumandos, el éxito de los niños varía en función del grado de abstracción de los mismos, de modo que cuando la situación es totalmente concreta como acontece cuando los cardinales de los dos sumandos se representan mediante objetos reales o dibujados (II y IV), entonces se facilita la resolución de la tarea aditiva fundamentalmente en los preescolares $(87,92 \%)$, tal como se confirma en otros trabajos (Carpenter et al., 1981; Riley, 1981; Riley et al., 1983; Steffe et al., 1982; etc.). Cuando la situación es mixta, es decir, cuando sólo uno de los sumandos se simboliza con un guarismo, tal como ocurre en las pruebas I y III, se hace más compleja la solución del problema planteado $(47,335 \%$ en los niños pequeños), que alcanza su máxima dificultad al representar ambos sumandos con guarismos (prueba V) (37,33\% en los preescolares). Por 
otra parte, las diferencias entre las pruebas II y IV son prácticamente nulas, debido a que si bien por un lado en la última prueba se facilita la manipulación de objetos, por otro en la II se simplifica la separación de los objetos contados con respecto a los no contados, evitándose mejor el contar dos veces un mismo objeto, o no contarlo en absoluto. En este sentido, algunos autores (Carpenter y Moser, 1982; Ibarra y Lindvall, 1971; Steffe y Johnson, 1971; etc.) han encontrado también que la manipulación o no de las ayudas resulta irrelevante para los niños. Y en cuanto a la situación mixta, el modo de representar el segundo sumando tiene mayor relevancia que en el caso del primero, ya que la prueba III resulta más difícil que la I, sobre todo para los sujetos más jóvenes, tal como puede observarse en la tabla I. Esto se debe, probablemente, a que estos niños suelen emplear la estrategia de contar todo, es decir, los dos sumandos; de modo que inician el conteo de las unidades que representan el primer sumando, y una vez alcanzado éste, prosigue la secuencia numérica con el conteo de los círculos, que simbolizan el segundo sumando. Ahora bien, cuando el segundo sumando es un guarismo, entonces resulta complejo continuar la secuencia numérica producida al contar el primer sumando, de modo que los niños tienden, bien a contar el guarismo como si fuera una unidad más, bien a iniciar por el uno el conteo del segundo sumando, rompiendo en este caso la secuencia numérica anterior. La solución más fácil, raramente empleada por los preescolares, consiste en aplicar la commutatividad, comenzando el conteo por el segundo sumando. $\mathrm{O}$ bien, representar primeramente el guarismo del segundo sumando mediante unidades concretas (por ejemplo, dedos de la mano), prosiguiendo después el conteo de ambos sumandos.

TABLA III

Porcentajes de tipos de estrategias más utilizadas en niños de $2 .^{\circ}$ de preescolar. (C: contar todo a partir del primer sumando; D: contar todo a partir del segundo sumando)

\begin{tabular}{|c|c|c|c|}
\hline \multirow[t]{2}{*}{ Pruebas } & \multicolumn{3}{|c|}{ Problemas } \\
\hline & $5+12$ & $11+4$ & $8+8$ \\
\hline I & $D(40)$ & $X(44)$ & $C(44)$ \\
\hline II & $C(84)$ & $C(84)$ & $C(83,33)$ \\
\hline III & $C(60)$ & $C(64)$ & $C(64)$ \\
\hline IV & $C(84)$ & $C(88)$ & $C(84)$ \\
\hline V & $C(40)$ & $C(72)$ & $C(60)$ \\
\hline
\end{tabular}

Las estrategias empleadas por los niños en la resolución de los problemas aditivos planteados dependen al menos de la edad o escolaridad de los sujetos, de la magnitud y posición de los sumandos, y del modo de representar estos sumandos. Así, mientras que los preescolares utilizan preferentemente la estrategia de contar todo, sea cual fuerte en general la prueba propuesta; los escolares de $10^{\circ}$ de EGB seleccionan la estrategia sobre todo en función de los sumandos. En este sentido, cuando el segundo de los su- 
TABLA IV

Porcentajes de tipos de estrategias más utilizadas en niños de $10^{\circ}$ de EGB. (A: contar todo a partir del primer sumando; $B$ : contar a partir del segundo sumando)

\begin{tabular}{cccc}
\hline Pruebas & $5+12$ & $\begin{array}{c}\text { Problemas } \\
11+4\end{array}$ & $8+8$ \\
\hline I & A (54,17) & A (84) & A (68) \\
\hline II & B (60) & A (60) & F (36) \\
\hline III & B (56) & A (60) & F (40) \\
\hline IV & B (44) & A (64) & F (52) \\
\hline V & B (48) & A (60) & F (52)
\end{tabular}

mandos es mayor que el primero (problema A) entonces la estrategia más empleada consiste en contar todo a partir del primer sumando, en el caso de los niños pequeños, mientras que los mayores usan más bien el procedimiento de contar a partir del cardinal del $2 .^{\circ}$ sumando. Sólo en la prueba I ambos grupos de sujetos cambian la mayoría de las veces su estrategia, sea para contar todo empezando por el segundo sumando (grupo I), sea con el fin de contar a partir del primer cardinal (grupo II). Por tanto, los preescolares suelen contar todo, en general, a partir del primer sumando, ayudándose con los dedos para representar los guarismos existentes en los problemas propuestos. Los escolares normalmente aplican primero la propiedad de la conmutatividad y, después, cuentan a partir del segundo sumando, es decir, cuenta el primer sumando, que es el más pequeño, utilizando los dedos de una mano. Pero conviene resaltar que en la prueba I, los pequeños también aplican la propiedad conmutativa debido probablemente a que les resulta más fácil y más directo iniciar el conteo por los círculos dibujados, para seleccionar después los dedos que representan el otro sumando, y contarlos a continuación, prosiguiendo la secuencia numérica detenida durante escasos segundos. De aquí que este modo de proceder lo manifieste prácticamente sólo en los tres problemas de esta prueba I y no en las siguientes pruebas, como sería de esperar, si la razón fuese la ubicación del sumando mayor. Este efecto del modo de representar los sumandos es notorio excepcionalmente en los niños mayores ya que sólo en la prueba I se comportan de manera similar independientemente del tipo de problema planteado. Sin embargo, estos niños parten del cardinal del primer sumando, que es un guarismo, para proseguir contando los círculos que representan el segundo sumando, evitando de este modo la compleja tarea de representar con los dedos uno de los sumandos.

En los problemas B y C, los preescolares utilizan, en general, la estrategia de contar todo, iniciándose la enumeración con el primer sumando, tal como se ha visto anteriormente. En cambio, los escolares prefieren emplear la estrategias de contar a partir del cardinal del primer sumando en el problema $\mathrm{B}$, mientras que prefieren solventar el problema $\mathrm{C}$ (el «doble») memorísticamente, excepto en la prueba I, como ya hemos referido. En consecuencia, los niños pequeños se limitan prácticamente a aplicar la estrategia de contar todo independientemente del tipo de problema y de la 
prueba propuestos; manifestando que sus recursos estratégicos son aún muy limitados. En cambio, los escolares suelen cambiar de estrategia en función del tipo del problema presentado, afectándoles menos el modo de representar los dos sumandos. Así en el problema B cuentan a partir del cardinal del primer sumando, representado el segundo sumando mediante dedos, cuando se trata de un guarismo. Y en el caso del «doble" (problema C) lo resuelven, en general, de memoria; ya que es bien conocido (Broen y Parkman, 1972; Svenson, 1975; Svenson y Broquist, 1975) que los niños suelen aprender antes las operaciones con el «doble».

Por otra parte, la mayoría de los errores cometidos por el grupo de los pequeños aparecen en las pruebas III y $\mathrm{V}$, empleando aquí también la estrategia de contar todo empezando por el primer sumando. Una vez más se muestra la dificultad de estos niños para representar los guarismos mediante dedos; ya que el uso de la estrategia mencionada suele conducir más bien a la solución correcta, tal como ocurre en las pruebas II y IV. En cambio, la estrategia de contar todo, empezando por el segundo sumando, es la que más errores produce en la prueba I. Los sujetos de $1 .^{\circ}$ de EGB cometen pocos errores apareciendo éstos sobre todo en las pruebas III y V, cuando los niños representan alguno o los dos sumandos mediante dedos, tal como acontece al usar en estos casos las estrategias de contar todo, o de contar a partir del primer sumando. De nuevo surge el problema de la representación, se trata de un tema de los procesos que conducen a la representación, extremadamente interesante, pero que rebasa obviamente los objetivos empíricos de este trabajo.

\section{CONCLUSIONES}

El éxito de los niños varía notoriamente en función de la edad y grado de escolaridad de las muestras; de modo que los preescolares obtienen rendimientos significativamente inferiores a los de los escolares, que alcanzan en general cotas muy elevadas. Pero esto no quiere decir que la respuesta correcta suponga que los niños, sobre todo los pequeños, comprendan perfectamente todo el alcance y significado de la operación de adición. Al contrario, entendemos que esta comprensión tiene niveles, que se realiza a lo largo del tiempo, y que sólo la consecución de una cierta madurez cognitiva posibilita el uso de estrategias variadas en la solución de estos problemas, como hemos visto. Por otra parte, la magnitud y ubicación de los sumandos afecta sobre todo a los niños pequeños, que llegan a fracasar casi en el $50 \%$ de los ensayos cuando el cardinal mayor se sitúa en el segundo sumando. Ello prueba que estos sujetos no poseen aún un amplio repertorio de estrategias, que les permitiría aplicar convenientemente la propiedad conmutativa, tal como suelen hacer los mayores. En cambio, los efectos positivos del «doble» apenas o nada se manifiestan en este caso, debido probablemente al uso de un número excesivamente alto.

Con respecto al modo de representar los sumandos, el éxito infantil depende claramente del grado de abstracción de los mismos, sobre todo en los preescolares, que alcanzan sus mejores resultados cuando la situación es totalmente concreta, es decir, cuando se representan los dos sumandos 
mediante objetos reales o pintados. En cambio, descienden notoriamente estos rendimientos en la condición simbólica, cuando ambos sumandos son guarismos; para obtener puntuaciones intermedias en la situación mixta: uno de los sumandos es un guarismo, mientras que el otro se representa mediante objetos. Este proceso de abstracción gradual resulta extremadamente interesante, a nuestro entender, ya que permite introducir más adecuadamente a los niños en el ámbito de lo simbólico, que domina ostensiblemente en nuestro sistema educativo. En consecuencia, el aprendizaje de la adición debería realizarse de manera escalonada, de modo que al principio se representarían ambos sumandos mediante objetos concretos, después se simbolizaría el primero de los sumandos por un guarismo, para en una etapa posterior representar mediante guarismos sólo el segundo sumando, y terminar en una situación completamente simbólica, constituida por números.

Las estrategias empleadas en la solución de los problemas aditivos propuestos son función tanto de la edad de los niños, como de la magnitud y ubicación de los sumandos, y de la representación de los mismos. Así, mientras que los preescolares utilizan en general la estrategia de contar todo, independientemente de la prueba presentada, los escolares de $1 .^{\circ}$ de EGB eligen su estrategia en función sobre todo de los sumandos. Raramente intentan los pequeños el uso de otras estrategias, y cuando lo hacen suelen fracasar en la realización de la tarea propuesta, aunque no siempre. Tal es el caso, por ejemplo, en la prueba I, ya que no pocos niños cuentan todo, pero aquí lo hacen empezando por el segundo sumando, esbozando lo que después denominaremos con exactitud la propiedad conmutativa. Este cambio de procedimiento, que significa sin ninguna duda un proceso importante en la comprensión de la operación aditiva, se ve facilitado por el modo de representar los sumandos, tal como ocurre en la mencionada prueba I, que constituye un modelo eficaz para incitar e introducir a los niños en la adquisición de a commutatividad. En cambio, el repertorio de estrategias de los mayores es más variado, realizando la elección en función de su competencia cognitiva y de la rapidez para llegar a la solución del problema. Así, en el caso del "doble" responden frecuentemente de memoria, mientras que cuando el segundo sumando es mayor que el primero, emplean preferentemente la propiedad conmutativa.

Finalmente, los errores cometidos por los sujetos suelen ser instructivos, no sólo desde el punto de vista educativo, sino también como reveladores de los procesos que conducen a la adquisición del concepto estudiado. En nuestro caso, los errores se refieren sobre todo a dificultades de representación por parte de ambos grupos, aunque principalmente en los preescolares.

\section{Referencias}

ASHCRAFT, M (1982). The develoment of mental arithmetic: A chronometric approach. Developmental Review, 2, 213-236.

AsHCRAFT, M. (1983). Procedural knowledge versus fact retrieval in mental arithmetic: A reply to Baroody. Developmental Review, 3, 231-235.

BAROODY, A. (1983). The development of procedural knowledge: An alternative explanation for chronometric trends of mental arithmetic. Developmental Review, 3, 225-230. 
BERmejo, V., y LAGO, M. O. (en prensa). El aprendizaje de las matemáticas. Estado actual de las investigaciones. Papeles del psicólogo.

BERMEJO, V., y RODRÍGUEZ, P. (en prensa). Estructura semántica y estrategias infantiles en la solución de problemas verbales de adición. Infancia y Aprendizaje.

BRown, A. L., y CAMPIONE, J. C. (1986). Psychological theory and the study of learning disabilities. American Psychologist, 14, 1059-1068.

BROWN, J. S., y BURTON, R. R. (1978). Diagnostic models for procedural bugs in basic mathematical skills. Cognitive Science, 2, 155-192.

BROWN, J. S., y VANLEHN, K. (1980). Repair theory: A generative theory of bugs in procedural skills. Cognitive Science, 4, 379-426.

BROWN, J. S., y VANLEHN, K. (1982). Toward a generative theory of abugs". En T. Carpenter, J. Moser y T. Romberg (eds.), Addition and subraction: A cognitive perspective. Hillsdale, N. Jersey: Lawrence Erlbaum Assocites.

BuCHINGHAM, B. R., y MAACLATCHY, J. (1930). The number abilities of children when they enter grade one. En 29th Yearbook of the National Society for the Study of Education. Bloomington, Ind.: Public School Publishing.

CARPENTER, T., y MOSER, J. (1982). The development of addition and subtraction problemsolving skills. En T. Carpenter, J. Moser y T. Romberg (eds.), Addition and subtraction: Developmental perspective. Hillsdale, N. Jersey: Lawrence Erlbaum Associates.

CARPENTER, T., y MOSER, J. (1983). The adquisition of additon and subtraction concepts. En R. Lesh y M. Landau (eds.). Acquisition of mathematic conceptos and processes. Nueva York: Academic Press.

CARPENTER, T.; HieberT, J., y MOSER, J. (1983). The effect of instruction on children's solutions of addition and subtraction word problemas. Educational Studies in Matbematics, 14, 55-72.

CASE, R. (1978). Piaget and beyond: Toward a develpmentally based theory and technology of instruction. En R. Glaser (ed.), Advances in instructional psychology. Hillsdale, N. Jersey: Erlbaum.

CASE, R. (1982). General developmental influences on the acquisition of elementary concepts and algorithms in arithmetic. En T. Carpenter, J. Moser y T. Romberg (eds.), Addition and subtraction: A cognitive perspective. Hilldale, N. Jersey: Lawrence Erlbaum Associates.

DE CORTE, E., y VerschafFel, L. (1985). Beginning dirst graders" initial representation of arithmetic word problems. Journal of Matbematical Bebavior, 4, 3-21.

FUSON, K. (1982). The counting-on solution procedure: Analysis and empirical results. En T. Carpenter, J. Moser y T. Romberg (eds.), Addition and subtraction: A cognitive perpective. Hillsdale, N. Jersey: Lawrence Erlbaum Assocites.

FusON, K. (1984). More complexities in subtraction. Journal for Research in Matbematics Education, 15, 214-225.

FUSON, K. (1986). Teaching children to subtract by counting up. Journal for Research in Matbematics Education, 17, 172-189.

GIBB, E. (1965). Children's thinking in the process of subtraction. Journal of Experimental Education, 25, 71-80.

Groen, G., y PARKman, J. (1972). A chronometric analysis of simple addition. Psychological Review, 79, 329-343.

Groen, G., y POLL, M. (1973). Subtraction and the solution of open sentence problems. Journal of Experimental Child Psychology, 16, 292-302.

Jones, G.; Thornton, C., y ToOHEY, M. (1985). A Multi-Option Program for learning basic addition facts: Case studies and a experimental report. Journal of Learning Disabilities, $18,319-325$.

KNIGHT, F., y BEHRENS, M. (1928). The learning of the 100 addition combinations and the 100 subtraction combinations. Nueva York: Longmans, Green and Co.

RESNICK, L. (1983). A developmental theory of number undetstanding. En H. Ginsburg (ed.), The development of mathematical thinking. Nueva York: Academic Press.

Riley, M.; GREenO, J., y Heller, J. (1983). Development of children's problem-solving ability in arithmetic. En H. Ginsburg (ed.). The development of mathematical thinking. Nueva York: Academic Press.

SeCADA, W.; FusON, K., y HaLl, J. (1983). The transition from counting-all to counting-on in addition. Journal for Research in Mathematic Education, 14, 47-57.

SIEGLER, R., y RoBinSON, M. (1982). The development of numerical understanding. En $\mathrm{H}$. Reese y L. Lipsitt (eds.). Advances in child development and behavior. Nueva York: Academic Press.

StefE, L., y JoHnSON, D. (1971). Problem solving performances od first-grade children. Journal for Research in Matbematics Educations, 2, 50-64.

STEFFE, L.; THOMPSON, P., y RiCHARDS, J. (1982). Children's counting in arithmetical problem solving. En T. Carpenter, J. Moser y T. Romberg (eds.), Addition and subtraction: A cognitive perspective. Hillsdale, N. Jersey: Lawrence Erlbaum Associates.

SUPPES, P., y GROEN, G. (1967). Some counting models for first grade performance data on simple addition facts. En J. M. Scanduva (ed.). Research in mathematics education. Washington, DC: National Council of Teachers of Mathematics. 
SVEnson, O. (1975). Analysis of time required by children for simple additions. Acta Psychologica, 39, 289-302.

SVENSON, O., y BROQUIST, S. (1975). Strategies for solving simple additions problems. Scandinavian Journal of Psychology, 16, 143-151.

YOUNG, P., y O'SHEA, T. (1981). Errors in children's subtraction. Cognitiver Science, 5, 153-177.

\section{Extended summary}

Recent research on simple addition and subtraction word problems shed light on several factors that contribute differently to children's performance. We consider noteworthy the following aspects: The type of representation employed to show the addends; the size of the addends; and the placing of the larger and shorter addend.

Some researchers stressed the importance of establishing a graduation in the abstraction level when representing the addends in arithmetical problems (e.g., Case, 1982). This would allow us to reach a deeper understanding and an adequate level of automatic performance in children's behaviour, and then introduce more abstract materials. Despite the fundamental role played by this factor it was rarely taken as the main focus of research. Nevertheless, some empirical data suggested that the simultaneous use of concrete and abstract materials seemend to facilitate the access to more advanced resolution procedures (e.g., Fuson, 1986).

On the other hand, the effect of addend size was the topic of some research at the beginning of the century, but this type of study evolved to more sophisticated chronometric studies (e.g., Ashcraft, 1982). This research initially offered data such as the size of the sum as an index of complexity, later becoming, thanks to chronometric studies, more relevant in reference to the role played by the larger addend, the size of the second addend, etc.

In this research we analyse when and how the abstraction level and the size of the addends influence children's behaviour. In particular, we presented five situations that involved different abstraction degrees: I: number and circles; II: circles and circles; III: circles and number; IV; objects and objects; and V: number and number. Likewise, we posed three conditions related to the size of the addends: A: The first addend is smaller than the second one (i.e., $5+12$ ); B: the first addend is larger than the second one (i.e., $11+4$ ); and, $C$ : the first addend is the same as the second one (i.e., $8+8$ ). The children always had the opportunity to use their finger because the size of the smaller addend never went beyond ten.

These conditions were cross-matched to produce an experimental design 2 (Group I vs. Group II) $\times 3$ (Addends A vs. Addends B vs. Addends C) $\times 5$ (Mixed situations - tasks I and III- vs. concrete situations - tasks II and IV - vs. abstract situation - task V-) with repeated measures. Two group of children were chosen at random, 25 preschool children $(M: 5 ; 7)$ and 25 hildren from a $1 .^{\circ} \mathrm{EGB}$ class were tested individually.

The results showed that the main effects of the three factors were significant: group, addend representation, and addend size. Thus, there was a significant difference between both groups, the older children obtaining better results, not only quantitatively but also qualitatively. Besides, this 
difference was reflected both in the number of correct trials, and in the consistence showed along the different tasks.

With regard to the significance of the abstraction level factor, it is interesting to note that it is due to the notable differences between the purely concrete situations and the mixed or totally abstract ones; the former being easier to solve and for which a very high sucess rate was obtained. However, as will be seen more clearly in the paragraph devoted to the strategies employed along the tasks, within the mixed situation the effects of this factor seem more important when the higher degree of abstraction corresponds to the second addend (see, e.g., Carpenter and Moser, 1982; Ibarra and Lindvall, 1971; Steffe and Johnson, 1971, etc.). These types of representation and abstraction levels have a marked effect above all in the group of younger children.

As for the addend size factor, at least two reasons seem to account for its significance: 1) the shift from units to tens with $A$ and $C$ tasks (see, e.g., Resnick, 1983), and (2) the difficulty shown by children using their fingers in an organized manner manner to keep track of the uncounted addend. Younger children found it easier to solve problems in which the second addend was smaller than the first one, then problems with equal addends, and, finally, problems where the second addend was larger than the first one.

The last important aspects refers to the choices of strategy. Preschool children always counted everything, regardless of the characteristics of a particular task. The reiteration of this strategy may be due either to the fact that these children didn't know the commutative law or the irrelevance order principle, which is simpler than the law, or because they were not used to managing large addends. This latter possibility has nothing to do with their ability to recite (or even count) numbers up to twenty, because all the subjects were able to do so. On the contrary, the older children employed several strategies depending not so much on the addend representation type as on the addend size factor. So, when the addends were $5+12$, they employed the count from larger strategy, and counted on from the first addend when $11+4$ were the problem addends. Finally, this group showed the ability to use «number facts».

In conclusion, we consider it important to take advantage of this «spontaneous» hierarchization based on the difficulty in accurately carrying out the resolution plan of the problem, above all, for its relevance to teaching arithmetical concepts and symbolisms in elementary school. 